NOTE

\title{
The photo-electric current in laser-Doppler flowmetry by Monte Carlo simulations
}

\author{
Tiziano Binzoni ${ }^{1,2}$, Terence $S$ Leung $^{3}$ and Dimitri Van De Ville ${ }^{4}$ \\ ${ }^{1}$ Département des Neurosciences Fondamentales, University of Geneva, Geneva, Switzerland \\ ${ }^{2}$ Département de l'Imagerie et des Sciences de l'Information Médicale, University Hospital, \\ Geneva, Switzerland \\ ${ }^{3}$ Department of Medical Physics and Bioengineering, University College London, London, UK \\ ${ }^{4}$ Biomedical Imaging Group, Ecole Polytechnique Fédérale de Lausanne (EPFL), Lausanne, \\ Switzerland \\ E-mail: Tiziano.Binzoni@unige.ch
}

Received 7 April 2009, in final form 8 June 2009

Published 30 June 2009

Online at stacks.iop.org/PMB/54/N303

\begin{abstract}
Monte Carlo (MC) simulations significantly contributed to a better understanding of laser-Doppler flowmetry (LDF). Here it is shown that the data obtained from standard MC simulations can be reinterpreted and used to extract more information such as the photo-electric current $(i(t))$. This is important because $i(t)$ is the starting point for evaluating any existing or new algorithm to be used in LDF instrumentation. This circumvents the tedious procedure of generating a specific model (often approximated if possible at all) each time a different algorithm is considered. By a series of tutorial examples, the influence of various parameters is investigated, e.g. sampling rate, total acquisition time and dc filtering. These cases also demonstrate the fundamental role played by the photons' random phase in the shaping of the LDF signal. In particular, it is demonstrated by MC simulation that when the number of photon-moving red blood cell interactions is too low, then the Siegert relation that exists between the field and photo-electric current autocorrelation functions does not hold. This is an important point because the validity of the Siegert relation is implicitly admitted in the majority of the classical analytical models for the autocorrelation function in LDF (the classical MC approach does not allow one to study this problem). The proposed method and examples could stimulate new ideas and help the scientific community develop, test and validate new approaches in LDF.
\end{abstract}

(Some figures in this article are in colour only in the electronic version)

\section{Introduction}

The frequency shift generated by the interaction of near-infrared light with moving red blood cells has been successfully exploited over the last 30 years to assess, non-invasively, blood 
velocity and flow in human tissues. The development of performant detectors such as CCD and CMOS has also allowed one to conceive real-time tissue blood perfusion imagers (Serov et al 2005, Briers 2007, Draijer et al 2009, Raabe et al 2009). On the other hand, the utilization of high-sensitive avalanche photodiodes or single photon counters combined with digital autocorrelators has opened the way to the investigation of tissue regions situated at more than $1 \mathrm{~cm}$ deep under the skin surface (for general reviews, see Leahy et al (1999), Briers (2001, 2007), Humeau et al (2007), Vennemann et al (2007)).

These techniques are based on analytical models that allow one to relate the acquired timedependent photo-electric current $(i(t))$ to tissue blood velocity- or flow-related quantities. The models become very different depending on the specific hardware implementations and on the morphology and optical properties of the observed tissues. Further on, the experimentalist and the theoretician face the problem of testing their validity, a mandatory step when dealing with laser-Doppler flowmetry (LDF) research and development.

Two methods are usually considered for LDF testing and calibration: (1) direct measurements on real tissue preparations, synthetic tissue phantoms or motility standards (Nilsson et al 1980, Smits et al 1986, Ahn et al 1987, Fairs 1988, Obeid 1993, Liebert et al 1995, 1999, Steenbergen and de Mul 1998a, 1998b, Leahy et al 1999) and (2) testing of the analytical models alone on numerically simulated data (Stern 1985, Koelink et al 1994, de Mul et al 1995, Boas 1996, Kienle 2001). This note deals with the second approach.

Many models are based on approximate analytical expressions for the power spectrum $\left(P_{i}(\omega)\right)$ or the normalized autocorrelation function $\left(g_{i}^{(2)}(\tau)\right)$ of $i(t) . P_{i}(\omega)$ and $g_{i}^{(2)}(\tau)$ are extremely important because they allow one to extract the tissue blood velocity or the flow information (Bonner and Nossal 1981). The 'gold standard' to test these analytical models is based on Monte Carlo (MC) simulations of the light transport into the tissue (Wilson and Adam 1983) with the added capability of taking the laser-Doppler effect into account (Stern 1985, Koelink et al 1994, de Mul et al 1995, Boas 1996, Kienle 2001). The 'classical' MC approach allows one to directly generate, in a numerical way, the key measures $P_{i}(\omega)$ or $g_{i}^{(2)}(\tau)$ without computing $i(t)$.

Today, LDF hardware is pushed to its limits, e.g., for full-field LDF (Serov et al 2002, 2005, Serov and Lasser 2005, Binzoni and Van De Ville 2008). This imposes the development of alternative solutions that allow one to process $i(t)$ in a different and more efficient manner. Even hardware implementations for the calculation of $P_{i}(\omega)$ or $g_{i}^{(2)}(\tau)$ become too slow and expensive as the operation must be applied for thousands of pixels simultaneously and in real time. Moreover, obtaining $i(t)$ in a simulation setting would allow us to better understand the physical parameters that influence the LDF signals but are not related to blood flow and thus appear as unwanted information (e.g. random phase contributions accumulated by the photons during their propagation into the tissue).

Consequently, direct access to $i(t)$ for simulation may become an important issue. The ability to numerically generate $i(t)$ should allow us to test and validate new ideas and to develop innovative LDF hardware. For these reasons, the aim of this note is to show that $i(t)$ can be obtained by extending/adapting the MC approach. The proposed method implies only a slight modification of the 'classical' MC approach as it exists for direct generation of $P_{i}(\omega)$ (see e.g. Soelkner et al (1997)).

\section{Material and methods}

In this section, we explain how $i(t)$ can be obtained by means of MC simulation. To that aim, we first summarize the 'classical' MC procedure that leads directly to $P_{i}(\omega)$. We will point out that a mathematical constraint on the photons' random phase plays a key role when 
assessing $i(t)$. Next, a series of tutorial examples concerning $i(t)$ is introduced and their practical importance is demonstrated.

\subsection{Reorganizing data obtained from 'classical' Monte Carlo simulations}

The 'classical' MC code for LDF simulations basically generates $N_{\text {packet }}$ couples $\left(W\left(\Delta \omega_{m}\right), \Delta \omega_{m}\right)\left(m=1,2, \ldots, N_{\text {packet }}\right)$, where $W\left(\Delta \omega_{m}\right) \in[0,1]$ is the fraction of photons within a photon packet that has experienced a given laser-Doppler shift $\Delta \omega_{m}$ and that has reached the photo-detector. The positive integer $N_{\text {packet }}$ corresponds to the number of photon packets launched in the simulation. When a photon packet does not reach the photo-detector, it is stored as the couple $\left(W\left(\Delta \omega_{m}\right), \Delta \omega_{m}\right)=(0,0)$. The laser-Doppler frequency shift, $\Delta v_{m}(\mathrm{~Hz})$, is expressed as an angular frequency: $\Delta \omega_{i}=2 \pi \Delta v_{m}$. In practice, $\left(W\left(\Delta \omega_{m}\right), \Delta \omega_{m}\right)$ couples are stored under the form of a histogram; i.e., all $W\left(\Delta \omega_{m}\right)$ with $\Delta \omega_{m} \in\left[n \delta \omega-\frac{\delta \omega}{2}, n \delta \omega+\frac{\delta \omega}{2}\right]$ are summed and associated with the $n$th bin and centred at the frequency $n \delta \omega$, where $\delta \omega$ is the bin width and $n \in \mathbb{Z}$ is the bin index.

The key point in the present work is that the complete $\left(W\left(\Delta \omega_{m}\right), \Delta \omega_{m}\right)$ data are stored as an $N_{\text {packet }} \times 2$ matrix; the histogram representation can always be build afterwards from this matrix for any chosen $\delta \omega$ value. This is the only modification to the core of the MC code with respect to the 'classical' method. The MC code utilized in the present work has already been presented in Binzoni et al (2008a, 2008b) but any other code can be adapted in a similar way.

\subsection{Analytical expressions for the electric field and $i(t)$}

A huge amount of work was done in the past to build a model allowing one to reliably describe $i(t)$. Different approaches to the problem have been investigated, going from semi-classical (e.g. Mandel et al (1964), Lamb and Scully (1969)) to the quantum electrodynamics (QED) theory (e.g. Glauber (1965), Nussenzveig (1973)) of photo-electric signals. Fortunately, in the context of biomedical LDF applications, $i(t)$ can be simplified to a well-known expression (Mandel et al 1964) that is usually written as (Forrester 1961, Cummins and Swinney 1970, de Mul et al 1995)

$$
i(t)=e \sigma 2 \mathcal{E}(t) \mathcal{E}(t)^{*},
$$

where $\mathcal{E}(t)$ is the (complex) electric field, $e \simeq-1.602 \times 10^{-19} \mathrm{~s}$ A is the electronic charge and $\sigma$ is a suitably defined quantum efficiency. For the present purpose, $\mathcal{E}(t)$ can be written in a general manner as

$$
\mathcal{E}(t)=\int_{0}^{\infty}\left[\beta^{\frac{1}{2}} \tilde{E}(\omega) \mathrm{e}^{\mathrm{i} \Phi(\omega)}\right] \mathrm{e}^{-\mathrm{i} \omega t} \mathrm{~d} \omega,
$$

where $\Phi(\omega)$ is the wavelength-dependent random phase and $\beta$ is the constant related to the degree of spatial coherence used in laser-Doppler theory which explains the absence of explicit spatial variables in the equations. Note that $\tilde{E}(\omega)$ is real-valued. Equation (1) implicitly takes into account the 'filter effect' of the photo-detector that is not capable of detecting the high end of optical frequencies, $\omega=\omega_{0}+\Delta \omega, \omega_{0}$ represents the laser frequency and $\Delta \omega$ the Doppler shift. Therefore, only the low beating frequencies, $\Delta \omega$, contribute to $i(t)$. Note that due to the presence of $\Phi(\omega), i(t)$ is a time-dependent random variable. Due to the form of equation (1), $i(t)$ has only positive real values by definition.

Like equation (1), equation (2) is also a simplified model but, in this case, part of the simplifications is dictated by the MC approach. The MC approach considers that each photon, after interaction with a scatterer, behaves as a plane wave travelling in a given direction. These photon waves do not interact inside the tissue, i.e. there are no interference phenomena. 
Moreover, the photo-detector is sensitive only to the squared norm of the electric field. Intuitively, this explains why the complex electric field $\mathcal{E}(t)$ is defined as a scalar and the wave vector is not present in equation (2). The exact motivation of equations (1) and (2) is not trivial, but they form a well-accepted model in the classical LDF literature (Cummins and Swinney 1970).

The link between equation (2) and the MC data ${ }^{5}$ (see section 2.1) is simply made by observing that

$$
W(\Delta \omega) \propto \tilde{E}\left(\omega_{0}+\Delta \omega\right)^{2} .
$$

In other words, the weights computed with the MC simulation are proportional to $\tilde{E}\left(\omega_{0}+\Delta \omega\right)^{2}$ and in this note we replace $\tilde{E}\left(\omega_{0}+\Delta \omega\right)^{2}$ by $W(\Delta \omega)$.

Given $\beta, \tilde{E}(\omega)$ and $\Phi(\omega)$, it is possible to numerically evaluate (1) and (2). The parameter $\beta$ is typically set to 1 for the simulations, and this does not influence the conclusions of the present work. The function $\tilde{E}(\omega)$ can be obtained according to equation (3), whereas $\Phi(\omega)$ is a critical remaining unknown. In the following section, we will see that the necessary information allowing one to explicitly define $\Phi(\omega)$ can be obtained by studying the 'classical' procedure (Forrester 1961, de Mul et al 1995) permitting to directly derive $P_{i}(\omega)$ from the MC data.

In conclusion, if we assume for now that $\Phi(\omega)$ can be determined, one has potentially two possibilities: (i) directly compute $i(t)$ by using $\tilde{E}(\omega)$ (derived from the MC data) and $\Phi(\omega)$ and then apply any old or new signal processing technique directly to $i(t)$ (such is the case for a real LDF instrument) or (ii) directly obtain the analytical expressions, e.g., for $P_{i}(\omega)$ or $g_{i}^{(2)}(\tau)$ (Stern 1985, Koelink et al 1994, de Mul et al 1995, Boas 1996, Kienle 2001) and then include the MC-derived data in these expressions to obtain the explicit numerical solution; this is the 'classical' MC approach and does not necessitate the use of $\Phi(\omega)$ (see below).

We will see that even if both solutions are compatible, solution (i) represents the best approach because it is more general and precise than solution (ii); it is similar to the real LDF data treatment and has the potentiality of easily evaluating future new algorithms.

Equations (1) and (2) hold for any chosen velocity distribution for the red blood cells. The influence of the velocity is included in the optical spectrum $\tilde{E}\left(\omega_{1}\right)^{2}$ as well as any complex, multiple-tissue, geometrical shape describing the observed biological sample and introduced through the MC simulation.

\subsection{Definition of $P_{i}(\omega), g_{i}^{(2)}(\tau)$ and physical constraints on $\Phi(\omega)$}

We present a procedure to analytically obtain $P_{i}(\omega)$ from $i(t)$ that is similar to de Mul et al (1995); see their appendix. We highlight the main points that are important to this work.

2.3.1. Analytical expression for $P_{i}(\omega)$ and physical constraints on $\Phi(\omega)$. By the WienerKhintchine theorem, $P_{i}(\omega)$ can be expressed as (Cummins and Swinney 1970)

$$
P_{i}(\omega):=\left|\int_{-\infty}^{\infty} i(t) \mathrm{e}^{-\mathrm{i} \omega t} \mathrm{~d} t\right|^{2}=\int_{-\infty}^{\infty}\left[\int_{-\infty}^{\infty} i(t) i(t+\tau) \mathrm{d} t\right] \mathrm{e}^{-\mathrm{i} \omega \tau} \mathrm{d} \tau .
$$

By substituting equations (1) and (2) into equation (4) and by using the integral definition of the Dirac function, one obtains

5 In a more precise manner, the 'wave' vision (equation (2)) and the 'particle' vision (MC simulation, section 2.1) are linked by the QED theory (Cohen-Tannoudji et al 2004). 


$$
\begin{gathered}
P_{i}(\omega)=16 \pi^{2} \beta^{2} e^{2} \sigma^{2} \int_{0}^{\infty} \int_{0}^{\infty} \tilde{E}\left(\omega_{2}\right) \tilde{E}\left(\omega_{1}\right) \tilde{E}\left(\omega_{1}+\omega\right) \tilde{E}\left(\omega_{2}+\omega\right) \\
\times \mathrm{e}^{-\mathrm{i}\left[\Phi\left(\omega_{1}\right)-\Phi\left(\omega_{1}+\omega\right)\right]} \mathrm{e}^{\mathrm{i}\left[\Phi\left(\omega_{2}\right)-\Phi\left(\omega_{2}+\omega\right)\right]} \mathrm{d} \omega_{1} \mathrm{~d} \omega_{2} .
\end{gathered}
$$

As already explained by different authors (Cummins and Swinney 1970, Bonner and Nossal 1981, de Mul et al 1995), the essential point is that the second term on the rhs of equation (5) is non-vanishing only if

$$
\omega_{1}=\omega_{2} \Longrightarrow \Phi\left(\omega_{1}\right)=\Phi\left(\omega_{2}\right) .
$$

It must be noted that the equality constraint on the phase is not trivial because $\Phi(\omega) \in[0,2 \pi]$ represents a 'random variable'. This result comes from the fact that experimentally, $\Phi(\omega)$ 'decorrelates' a lot faster than the observed phenomenon. In practice, $\Phi(\omega)$ may reasonably be considered as a uniformly distributed random variable (but this is not a necessary condition). In other words, relation (6) indicates that if two photons reach the photo-detector with the same frequency value $\omega$, then they must also carry the same (random) phase value $\Phi(\omega)$.

The importance of the above result is twofold: (i) it allows one to include $\Phi(\omega)$ in equation (2) (and thus in $i(t)$ ) because it gives the missing information and (ii) it allows the use of the computer time-saving variance reduction technique (Wang et al 1995). The variance reduction technique consists in launching a series of photon packets and not single photons. By construction, photons of the same packet always carry the same frequency and have followed exactly the same path into the tissue at exactly the same time. The above observation tells us that these photons must also have the same phase. The fact that it is not necessary to define a different $\Phi(\omega)$ realization for each photon composing a photon packet and reaching the detector is essential to the variance reduction technique.

In the light of the above discussion it is easy to carry on the calculation, by restarting from equation (5), and obtain de Mul et al's (1995) exact analytical expression for the power spectrum:

$$
\begin{aligned}
P_{i}(\omega) & \approx P_{i}^{\infty}(\omega) \\
& = \begin{cases}16 \pi^{2} \beta^{2} e^{2} \sigma^{2}\left(\int_{0}^{\infty} \tilde{E}\left(\omega_{1}\right)^{2} \mathrm{~d} \omega_{1}\right)^{2} & \text { if } \omega=0 \\
16 \pi^{2} \beta^{2} e^{2} \sigma^{2} \int_{0}^{\infty} \tilde{E}\left(\omega_{1}\right)^{2} \tilde{E}\left(\omega_{1}+\omega\right)^{2} \mathrm{~d} \omega_{1} & \text { if } \omega \neq 0 .\end{cases}
\end{aligned}
$$

The weight of the Dirac at $\omega=0$ (first term on the rhs) assures the right value of the dc component.

2.3.2. Definition of $g_{i}^{(2)}(\tau)$. The definition for the normalized autocorrelation function of the photo-current is (Jakeman 1974)

$$
g_{i}^{(2)}(\tau):=\lim _{T \rightarrow+\infty} \frac{(2 T)^{-1} \int_{-T}^{T} i(t) i(t+\tau) \mathrm{d} t}{\left[(2 T)^{-1} \int_{-T}^{T} i(t) \mathrm{d} t\right]^{2}} .
$$

The field-normalized autocorrelation function, $g^{(1)}(\tau)$, can also be defined as

$$
g^{(1)}(\tau):=\lim _{T \rightarrow+\infty} \frac{(2 T)^{-1} \int_{-T}^{T} \mathcal{E}(t) \mathcal{E}(t+\tau)^{*} \mathrm{~d} t}{(2 T)^{-1} \int_{-T}^{T} \mathcal{E}(t) \mathcal{E}(t)^{*} \mathrm{~d} t} .
$$

For $\tau=0, g^{(1)}(\tau)$ is 1 for any $\mathcal{E}(t)$. 


\subsection{Plugging MC data into the analytical expressions for $i(t)$ and $P_{i}(\omega)$}

In section 2.2, we pointed out that the MC data can be used as an input for the analytical expressions of $i(t)$ or $P_{i}(\omega)$, i.e. by replacing $\tilde{E}\left(\omega_{0}+\Delta \omega\right)^{2}$ with $W(\Delta \omega)$. However, MC simulations generate only a series of weights for non-uniformly distributed frequency shifts, $\Delta \omega_{m}$. The non-uniformly sampled data can be used in two ways: (1) by directly considering the non-uniform $\omega$-sampling and then by solving the equations with the suitable non-uniform discrete algorithms or (2) by 'resampling' the function $\tilde{E}\left(\omega_{0}+\Delta \omega_{m}\right)^{2}$ by means of a histogram and then solving the equations for uniform $\omega$-sampling, which corresponds to the classical approach for $P_{i}(\omega)$. Next, both options are investigated. The former will be applied for the direct derivation of $i(t)$ and the latter for the classical derivation of $P_{i}(\omega)$. For simplicity, we will designate these as the 'direct method' and the 'histogram-based method', respectively.

2.4.1. Direct method. As already explained, in the MC simulations the continuous-domain function appearing in the square brackets of equation (2),

$$
X_{\text {cont }}(\omega):=\beta^{\frac{1}{2}} \tilde{E}(\omega) \mathrm{e}^{\mathrm{i} \Phi(\omega)},
$$

is obtained as a series of samples at different $\omega_{i}$ values and for this reason it can be rewritten as

$$
X_{\text {discr }}(\omega)=X_{\text {cont }}(\omega) \sum_{m=1}^{N_{\text {packet }}} \delta_{\mathrm{D}}\left(\omega-\omega_{m}\right)
$$

where $\delta_{\mathrm{D}}$ (.) is the Dirac delta function. The electric field $\mathcal{E}$ (equation (2)) can be expressed in the discretized form as

$$
\mathcal{E}_{\text {discr }}(t)=\beta^{\frac{1}{2}} \sum_{m=1}^{N_{\text {packet }}}\left[\tilde{E}\left(\omega_{m}\right) \mathrm{e}^{\mathrm{i} \Phi\left(\omega_{m}\right)}\right] \mathrm{e}^{-\mathrm{i} \omega_{m} t}
$$

and can be numerically implemented. As previously explained, the phase $\Phi(\omega)$ is defined as a set of $N_{\text {packet }}$ uniformly distributed independent random numbers $\Phi(\omega) \in[0,2 \pi]$ satisfying relation (6). From equations (1), (3) and (12), one obtains $i(t)$ as a function of the non-uniform MC data:

$$
i(t) \approx e \sigma 2 \beta\left|\sum_{m=1}^{N_{\text {packet }}}\left[\sqrt{W\left(\Delta \omega_{m}\right)} \mathrm{e}^{\mathrm{i} \Phi\left(\omega_{0}+\Delta \omega_{m}\right)}\right] \mathrm{e}^{-\mathrm{i} \Delta \omega_{m} t}\right|^{2}
$$

Note that $t$ in equation (13) is a continuous-domain variable and thus its values can be freely chosen depending on the needs of the simulation.

2.4.2. Histogram-based method. The 'classical' MC approach does not start from equation (13) but it directly combines MC data with the analytical solutions for $P_{i}(\omega)$ (equation (7)). Even in this case a non-uniform $\omega$-sampling can be used, but, to speed up the calculations, a regular histogram is usually constructed as explained in section 2.1. This approach assumes the existence of an underlying probability density function, $f(\Delta \omega)$, from which the $W\left(\Delta \omega_{m}\right)$ data are taken. The histogram is an estimator of the density $f(\Delta \omega)$. The histogram generated from the $W\left(\Delta \omega_{m}\right)$ data and evaluated at the (constant-spaced, $\delta \omega$ ) $\delta \omega_{n}$ values $\left(\delta \omega_{n}<\delta \omega_{n+1} ; \forall n \in\left\{1,2, \ldots, N_{\text {bin }}\right\}\right)$ is written in the present work as $\hat{h}_{W}\left(\delta \omega_{n}\right)$. The constant $N_{\text {bin }}$ is the number of bins in the histogram. 
Thus, the analytical expression for $P_{i}^{\infty}(\omega)$ (equation (7)) can now be written as $P_{i}^{\infty}\left(\delta \omega_{k}\right) \approx P_{\text {hist }}\left(\delta \omega_{k}\right)$

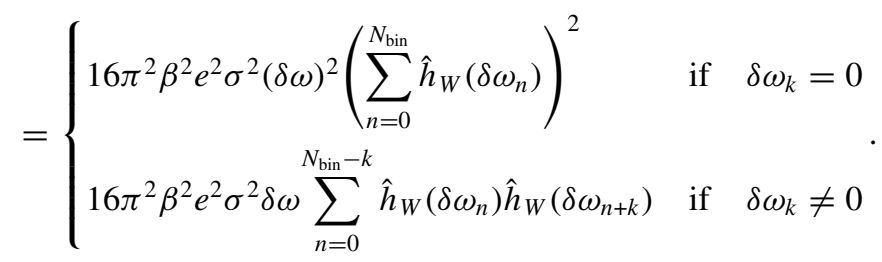

Equation (14) corresponds to the well-known classical numerical equation for $P_{\text {hist }}(\omega)$ (de Mul et al 1995), but where the $P_{\text {hist }}\left(\delta \omega_{k}\right)$ value for $\delta \omega_{k}=0$ is included.

\subsection{Data sets for the tutorial examples}

The set of MC data used in the tutorial examples has been computed on a PC cluster with eight nodes as in Binzoni and Van De Ville (2008). In summary, a virtual tissue phantom was represented by a homogeneous $2500 \times 2500 \times 2500 \mathrm{~mm}^{3}$ cube. LDF was a simple pointsource/detector configuration, with the source centred on and normal to one of the cube's surfaces. The cylindrical symmetry allowed one to treat the problem as a point source with an annular detector (75 $\mu \mathrm{m}$ width). The interoptode spacing $(0.5 \mathrm{~mm})$ was defined as the distance between the source and the middle point of the annular detector. The number of photon packets generated for one simulation was $N_{\text {packet }}=9 \times 10^{6}$. The absorption coefficient $\left(\mu_{\mathrm{a}}\right)$, the reduced scattering coefficient $\left(\mu_{\mathrm{s}}^{\prime}\right)$, the refractive index $(n)$, the anisotropy parameter $(g)$ and the wavelength $(\lambda)$ were set to $0.025 \mathrm{~mm}^{-1}, 0.5 \mathrm{~mm}^{-1}, 1.4,0.9$ and $800 \mathrm{~nm}$, respectively, for all the simulations. The refractive index for air was set to 1 . The physiological parameters were $P_{\text {move }} \in\{0.025,0.05,0.075,0.1,0.125,0.15\},\left\langle V_{\text {Brown }}^{2}\right\rangle^{1 / 2} \in\{1,2,3,4\} \mathrm{mm} \mathrm{s}^{-1}$ and $V_{\text {trans,x }} \in\{0,1,2,3,4\} \mathrm{mm} \mathrm{s}^{-1}$, where $P_{\text {move }}$ is related to the fraction of scatterers (red blood cells) moving inside the tissue and $\left\langle V_{\text {Brown }}^{2}\right\rangle^{1 / 2}$ is the root mean square of the red blood cells' velocity component due to the 'Brownian' motion (normal velocity distribution). $P_{\text {move }}$ can be seen as the probability for a photon packet to interact with a moving particle. The values here are for explanatory purposes and their range can account for different types of tissues. The vector $\vec{V}_{\text {trans }}=\left(V_{\text {trans }, x}, 0,0\right)$ represents a bulk translational velocity component of the red blood cells that is parallel to the surface of the cube where the optodes are situated. The model chosen for the blood flow is only an example. In fact, the behaviour of global mean blood flow may depend on the type of tissue and thus on the geometry of the related vascular network (see, e.g., the discussion section in Binzoni and Van De Ville (2008)).

The MC simulations performed with all the possible combinations of these parameters give a total of $120 \mathrm{MC}$ data sets allowing one to build 120 different $i(t)$ for a given $\Phi(\omega)$ random realization. The sampling frequency or total time duration of the digitalized $i(t)$ signal can obviously be chosen ad libitum. A supplementary set of MC data were also obtained for $P_{\text {move }}=0.30,\left\langle V_{\text {Brown }}^{2}\right\rangle^{1 / 2}=4 \mathrm{~mm} \mathrm{~s}^{-1}$ and $\vec{V}_{\text {trans }}=(4,0,0) \mathrm{mm} \mathrm{s}^{-1}$.

\section{Results}

We use the MC data and the expressions derived above to put together a number of tutorial examples illustrating $i(t), P_{i}(\omega)$ and $g_{i}^{(2)}(\tau)$ for different physiological and/or instrumental acquisition parameters. We also investigate the influence of $\Phi(\omega)$ on the shape of the LDF signals. The parameters $\beta, e$ and $\sigma$ were simply set to 1 (this does not change the present results). 

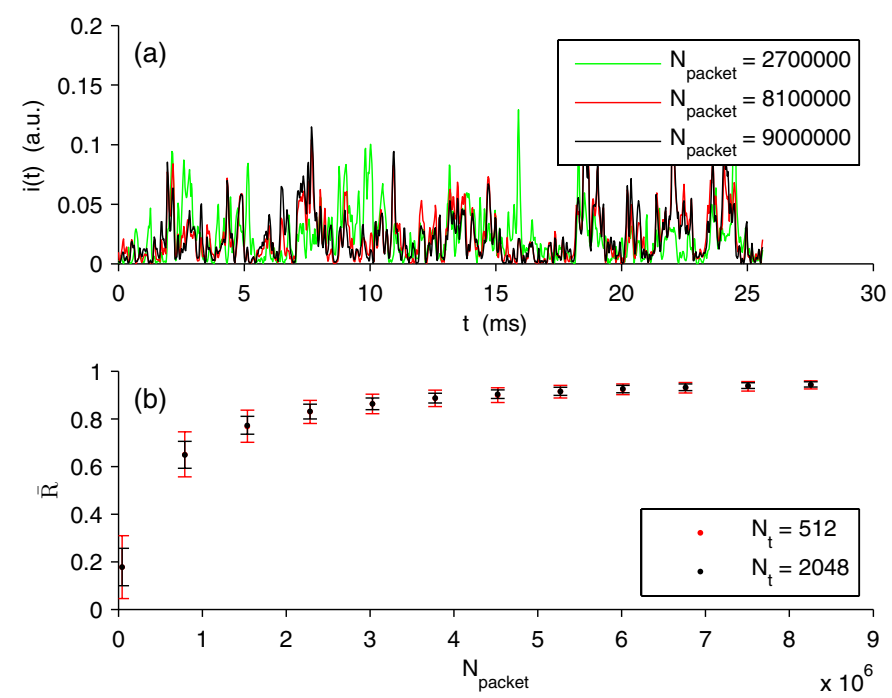

Figure 1. (a) Three versions of $i(t)$ for different $N_{\text {packet }}$ but common $\Phi(\omega)$ (see text). The physiological parameters were $P_{\text {move }}=0.15,\left\langle V_{\text {Brown }}^{2}\right\rangle^{1 / 2}=2 \mathrm{~mm} \mathrm{~s}^{-1}$ and $\vec{V}_{\text {trans }}=$ $(1,0,0) \mathrm{mm} \mathrm{s}^{-1}$. The sampling frequency was $40 \mathrm{kHz}$. (b) For the same MC data set, $i(t)$ is computed twice, once by using $N_{\text {packet }}$ and once by using $N_{\text {packet }}+746250$. The $R$ of the two $i(t)$ realizations was then obtained. The same operation was repeated for $120 \times 8 \times 12$ different MC data sets. The number of different $\Phi(\omega)$ realizations for each of the $120 \mathrm{MC}$ data sets is 8 . The number of points on the $N_{\text {packet }}$ axis is 12 . All the $i(t)$ computed with the same MC data set have a common $\Phi(\omega) . \bar{R}$ was then obtained as a function of $N_{\text {packet }}$. The sampling frequency was $40 \mathrm{kHz}$ and $N_{\mathrm{t}}$ is the number of sampling points. The vertical bars represent the standard deviation.

\subsection{Example: Monte Carlo generation of $i(t)$ and the role of $\Phi(\omega)$}

Figure 1(a) shows $i(t)$ computed by means of the direct method (equation (13)). These plots give a qualitative picture of the influence of the number of packets $\left(N_{\text {packet }}\right)$ on the derivation of $i(t)$. For large $N_{\text {packet }}, i(t)$ tends to converge (e.g., compare $N_{\text {packet }}=8100000$ and $\left.N_{\text {packet }}=9000000\right)$ and plots nicely overlap. For low $N_{\text {packet }}\left(N_{\text {packet }}=2700000\right), i(t)$ can in principle not be considered as a valid function for further calculations (see, however, the following sections). To allow the above comparison, the $i(t)$ curves were generated by using a common $\Phi(\omega)$. Specifically, if for two different choices of $N_{\text {packet }}$ the same frequency component at $\omega /(2 \pi)$ is used, then they have the same phase, $\Phi(\omega)$. Therefore, $i(t)$ that is built with larger $N_{\text {packet }}$ has statistically more (different) frequency components, which explains the difference between the curves in figure 1(a).

To better quantify the role of $N_{\text {packet }}$, in figure 1(b), we have computed the correlation coefficient $(R)$ of couples of $i(t)$ generated using different $N_{\text {packet }}$ values (also see the figure legend). For two $i(t)$ realizations to be perfectly equal, $R$ must be 1 . In figure 1(b), one can clearly observe that the mean $R(\bar{R})$ increases for larger $N_{\text {packet }}$ and that it converges to 1 . The standard deviation becomes also smaller for larger $N_{\mathrm{t}}$ (number of sampling points), while it decreases for larger $N_{\text {packet. }}$. This confirms the qualitative findings reported in figure 1(a). In fact, we will see in the following sections that the small difference of $R$ from 1 is due to the phase randomization by $\Phi(\omega)$, but that all the necessary information coming from the ('physiological') laser-Doppler shift is already completely contained in $i(t)$ for small $N_{\text {packet }}$ values. 

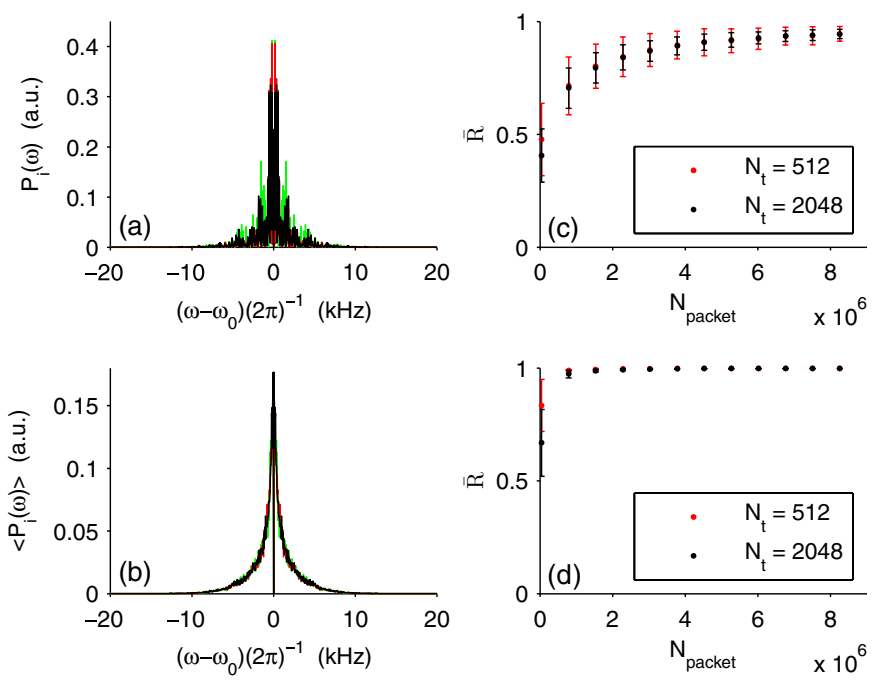

Figure 2. (a) $N_{\text {packet }}=2700000$ (green), $N_{\text {packet }}=8100000$ (red), $N_{\text {packet }}=9000000$ (black). $P_{i}(\omega)$ directly computed from three different $i(t)$ (where the dc offset has been subtracted). The MC data sets used to derive the three $i(t)$ were the same as in figure 1. The number of sampling points for $i(t)$ was $N_{\mathrm{t}}=1024$. To obtain comparable data, $i(t)$ was normalized by its energy. (b) Same as in panel (a) but in this case $\left\langle P_{i}(\omega)\right\rangle$ represents the mean of 100 spectra. The 100 different spectra were derived by using 100 different $\Phi(\omega)$ realizations (all the other parameters remaining constant). (c) For a given MC data set, $P_{i}(\omega)$ has been computed twice, once by using $N_{\text {packet }}$ and once by using $N_{\text {packet }}+746250$. The $R$ of the two $P_{i}(\omega)$ realizations was then obtained. The same operation is repeated for $120 \times 8 \times 12$ different data sets. The number of different $\Phi(\omega)$ realizations for each of the $120 \mathrm{MC}$ data sets is 8 . The number of points on the $N_{\text {packet }}$ axis is 12 . All the $P_{i}(\omega)$ computed with the same MC data set have a common $\Phi(\omega) . \bar{R}$ is then computed as a function of $N_{\text {packet }}$. The sampling frequency was $40 \mathrm{kHz}$ and $N_{\mathrm{t}}$ is the number of sampling points. The vertical bars represent the standard deviation. (d) Same as in panel (c) but for $\left\langle P_{i}(\omega)\right\rangle$.

\subsection{Example: Monte Carlo generation of $P_{i}(\omega)$ and the role of $\Phi(\omega)$}

Once $i(t)$ is determined by the direct method, the spectrum $P_{i}(\omega)$ can be derived as well (equation (4)). In figure 2(a), we show three $P_{i}(\omega)$ spectra obtained from different $i(t)$ (as in figure 1). Even in this case, the most apparent feature is the effect of the phase randomization. It is well known that in experimental reality, this problem is solved by taking the ensemble mean over a large number of $P_{i}(\omega)\left(\left\langle P_{i}(\omega)\right\rangle\right)$ (see figure 2(b)). Compared to $P_{i}(\omega),\left\langle P_{i}(\omega)\right\rangle$ is definitively less sensitive to $N_{\text {packet }}$ and the three curves perfectly coincide. The mean of a larger number of spectra further improves the coincidence (not shown). Consequently, averaging smooths out the random phase and the intrinsic laser-Doppler signal remains. Nevertheless, this does not signify the fact that the influence of $\Phi(\omega)$ has completely disappeared (see the following sections)!

By using the direct method for $i(t)$ and equation (4) to obtain $P_{i}(\omega)$, the $\bar{R}$ values for a set of different $P_{i}(\omega)$ and $\left\langle P_{i}(\omega)\right\rangle$ are shown in figures 2(c) and (d) respectively. It can clearly be seen from figure 2(d) that it is possible to use a very low number of photon packets $\left(N_{\text {packet }} \approx 1000000\right)$ to obtain a high-quality $\left\langle P_{i}(\omega)\right\rangle$ spectrum and this confirms, in a general manner, the qualitative findings from figure $2(\mathrm{~b})$. The standard deviation is smaller for large $N_{\mathrm{t}}$; additionally, it decreases for increasing $N_{\text {packet }}$.

It is useful to highlight the fact that the 'classical' histogram-based method (equation (14)) does not allow one to observe the influence of $\Phi(\omega)$ on the determination 

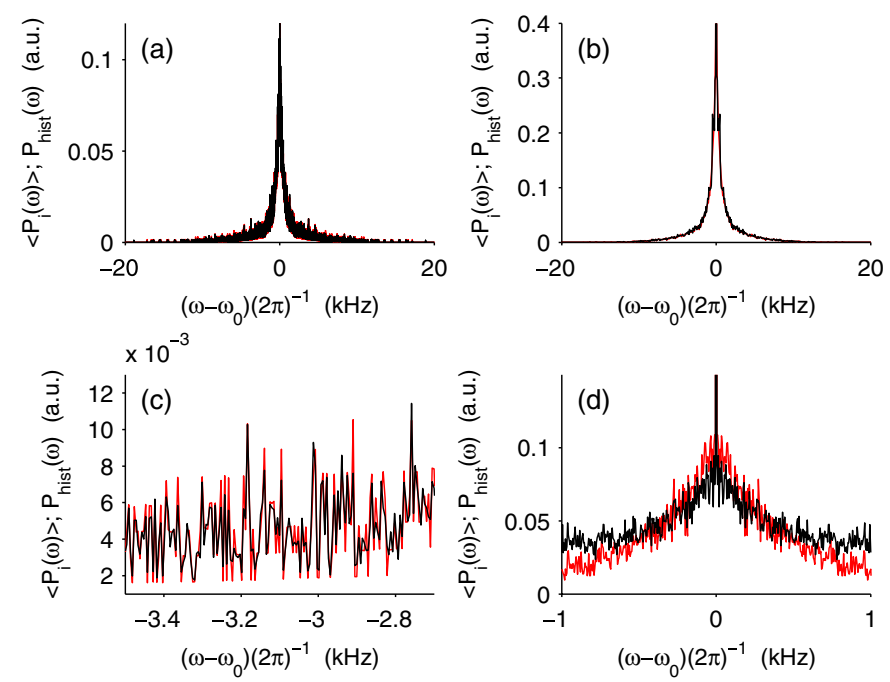

Figure 3. (a) $\left\langle P_{i}(\omega)\right\rangle$, computed by the direct method and equation 2. $P_{\text {hist }}(\omega)$, computed by using the histogram-based method (equation $(14)$ and $\delta \omega \approx 4.88 \mathrm{~Hz}$ ). $\left\langle P_{i}(\omega)\right\rangle$ (black) and $P_{\text {hist }}(\omega)$ (red) are reported together on the same axis. $\left\langle P_{i}(\omega)\right\rangle$ is the mean of 100 spectra. The photo-current $i(t)$ was sampled at $40 \mathrm{kHz}$ with $N_{\mathrm{t}}=8192$. The physiological parameters were $P_{\text {move }}=0.075,\left\langle V_{\text {Brown }}^{2}\right\rangle^{1 / 2}=3 \mathrm{~mm} \mathrm{~s}^{-1}$ and $\vec{V}_{\text {trans }}=(0,0,0) \mathrm{mm} \mathrm{s}^{-1} .\left\langle P_{i}(\omega)\right\rangle$ and $P_{\text {hist }}(\omega)$ are so similar that in practice, they cannot be distinguished. $N_{\text {packet }}=9000000$. (b) Same as in panel (a) but with $N_{\mathrm{t}}=512$ and $\delta \omega \approx 78.16 \mathrm{~Hz}$. (c) Zoom of panel (a) allowing one to see the matching between the two curves. (d) Same as in panel (a) but where the sampling frequency was $2 \mathrm{kHz}$ and $N_{\mathrm{t}}=410$ (same total time as in panel (a)).

of the LDF signals and that this makes up one of the advantages of the proposed approach.

As shown in figure 2, it is possible to remove the dc component from $i(t)$ by subtracting the mean value, similar to the procedure in LDF instrumentation. This operation has no real exact equivalent in the 'classical' histogram-based method because the zero-frequency bin of the histogram also contains frequencies different from zero (due to the bin size $\delta \omega>0$ ) and thus prevents precise elimination of only the dc component.

\subsection{Example: the influence of the sampling rate and finite time duration of $i(t)$}

In real LDF instrumentation, the signal $i(t)$ is sampled at a finite sampling rate during finite time $\left(T_{i}\right)$. Consequently, the quantities computed from $i(t)$, such as $P_{i}(\omega)$ or $\left\langle P_{i}(\omega)\right\rangle$, also depend on these 'non-physiological' parameters. This issue can be easily investigated using the present approach. In figures $3(\mathrm{a})$ and (b), we show an example where $\left\langle P_{i}(\omega)\right\rangle$ was derived from two different $i(t)$ with different $T_{i}(0.2048 \mathrm{~s}$ and $0.0128 \mathrm{~s}$ for figures $3(\mathrm{a})$ and (b), respectively) but the same sampling rate. The influence of the different $T_{i}$ values is clearly visible in the figure; the amplitude and bandwidth are different (this is equivalent to multiplying $i(t)$ by a 'square window' of duration $\left.T_{i}\right)$.

For the sake of completeness, $\left\langle P_{i}(\omega)\right\rangle$ was also computed by the histogram-based method $\left(P_{\text {hist }}\left(\delta \omega_{k}\right)\right.$; equation (14)). In this case it was necessary to adapt the bin width of the histogram because, to reproduce the same physical model as for the direct method, one needs $T_{i}=1 / \delta \omega$ by definition. Both methods give the same results. 


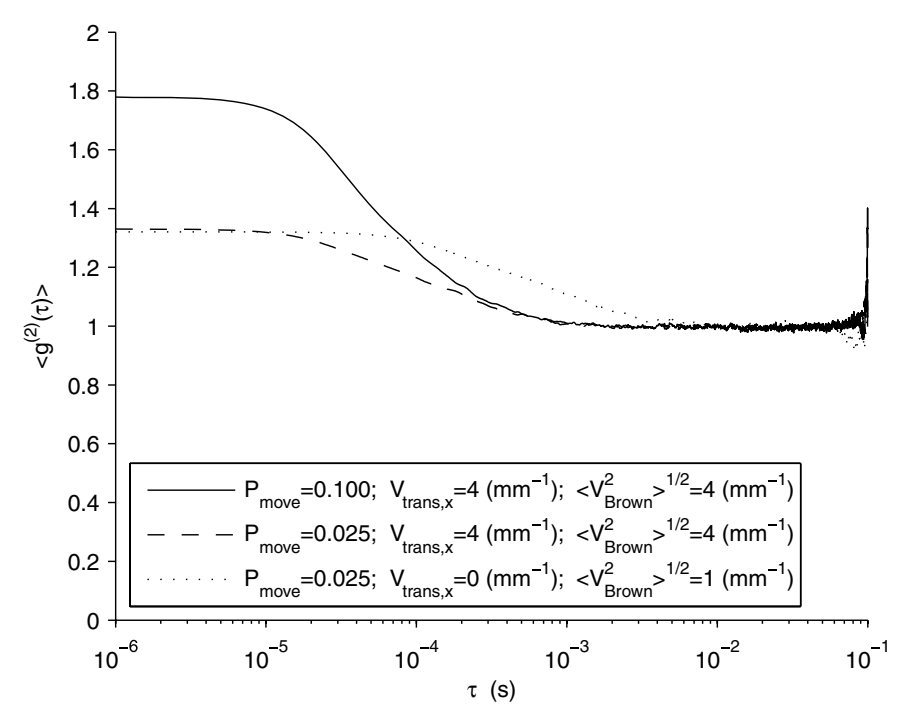

Figure 4. $\left\langle g_{i}^{(2)}(\tau)\right\rangle$ is the mean of $50 g_{i}^{(2)}(\tau)$ realizations. The photo-current $i(t)$ was sampled at $1 \mathrm{MHz}$ with $N_{\mathrm{t}}=100000$ and $N_{\text {packet }}=9000000$. The different $g_{i}^{(2)}(\tau)$ realizations were numerically estimated from $i(t)$ by using a standard unbiased algorithm of the autocorrelation function (reproducing equation (8)).

To better illustrate the similarity of the two methods (direct and histogram-based methods), figure 3(c) shows a zoom of figure 3(a). The key point is that the noisy aspect of the curves is not due to $\Phi(\omega)$. In fact, applying the histogram-based method is equivalent to taking the mean of an infinite number of $P_{i}(\omega)$ in the direct method to obtain $\left\langle P_{i}(\omega)\right\rangle$ (see e.g. figures 2(a) and (b)); in other words, $P_{\text {hist }}\left(\delta \omega_{k}\right) \approx\left\langle P_{i}\left(\delta \omega_{k}\right)\right\rangle$. As we have previously seen, the averaging procedure renders a smooth $\left\langle P_{i}(\omega)\right\rangle$. Actually, the remaining noise is produced by the finite $N_{\text {packet }}$ value, i.e. the intrinsic error of MC simulation that is reproduced in the same manner by both approaches.

Another typical issue in LDF instrumentation is the problem of undersampling (Binzoni and Van De Ville 2008). In figure 3(d), $\left\langle P_{i}(\omega)\right\rangle$ is shown when $i(t)$ is undersampled. The simulation was performed by using both the direct and the histogram-based methods. In the latter case, the effect of the undersampling was introduced by eliminating from the histogram frequency bins above the Nyquist frequency. This is obviously only a good approximation that does not hold in some cases (i.e. $\left.P_{\text {hist }}\left(\delta \omega_{k}\right) \neq\left\langle P_{i}\left(\delta \omega_{k}\right)\right\rangle\right)$. In fact, in figure 3(d), the curves of $\left\langle P_{i}(\omega)\right\rangle$ and $P_{\text {hist }}\left(\delta \omega_{k}\right)$ do not perfectly coincide and the exact solution is given by the direct method only (in black). This shows that the direct method takes into account the experimental acquisition parameters in a simple and natural manner.

\subsection{Example: Monte carlo generation of $g_{i}^{(2)}(\tau)$ and the Siegert relation}

One of the most interesting functions in $\operatorname{LDF}$ is $g_{i}^{(2)}(\tau)$. In the past, several analytical models have been derived with the aim to extract the physiological parameters from the $g_{i}^{(2)}(\tau)$ experimental measurements (see e.g. Pines et al (1990), Boas (1996), Skipetrov and Meglinski (1998), Binzoni et al (2006b)). A numerical model that allows one to test these analytical models and in particular that can take into account any blood velocity distribution and the role of $\Phi(\omega)$ appears to be extremely useful in this context. 


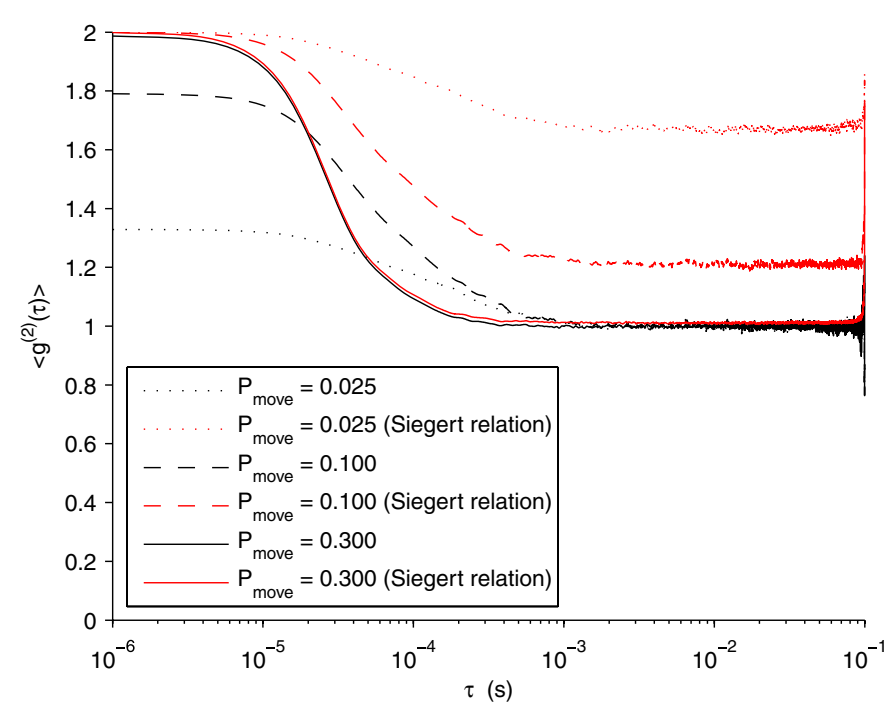

Figure 5. $\left\langle g_{i}^{(2)}(\tau)\right\rangle$ is the mean of $50 g_{i}^{(2)}(\tau)$ realizations. The photo-current $i(t)$ was sampled at $1 \mathrm{MHz}$ with $N_{\mathrm{t}}=100000, N_{\text {packet }}=9000000,\left\langle V_{\text {Brown }}^{2}\right\rangle^{1 / 2}=4 \mathrm{~mm}^{-1}$ and $\vec{V}_{\text {trans }}=(4,0,0)$ $\mathrm{mm} \mathrm{s}^{-1} \cdot g_{i}^{(2)}(\tau)$ was estimated in two ways: (1) directly from $i(t)$ by using a standard unbiased algorithm of the autocorrelation function reproducing equation (8) and (2) by using the Siegert relation and equation (15).

In figure 4, we show three examples of $\left\langle g_{i}^{(2)}(\tau)\right\rangle$ (direct method combined with equation (8)) obtained by considering different red blood cell velocities. The $\left\langle g_{i}^{(2)}(\tau)\right\rangle$ dependence on velocity clearly appears from the figure. However, the most interesting fact is that for increasing $P_{\text {move }}$ values (at $\left.\tau \approx 0\right),\left\langle g_{i}^{(2)}(\tau)\right\rangle$ also increases while remaining smaller than 2. This means that we are in a situation where the Siegert relation (Jakeman 1974), which links $g^{(1)}(\tau)$ and $g_{i}^{(2)}(\tau)$, does not hold $\left(\left\langle g_{i}^{(2)}(\tau)\right\rangle\right.$ is not equal to 2 for $\left.\tau \approx 0\right)$ and the simulation nicely reproduces this behaviour (see below and section 4$)$.

On the other hand, when $\tau \rightarrow+\infty$ then $\left\langle g_{i}^{(2)}(\tau)\right\rangle=1$. It is well known that this behaviour is due to the presence of the random $\Phi(\omega)$ implicitly included in $i(t)$. In fact, if one sets $\Phi(\omega)$ to a fixed value, then $\left\langle g_{i}^{(2)}(\tau)\right\rangle=0$ for $\tau \rightarrow+\infty$ (not shown). This phenomenon cannot be described by the classical MC simulations allowing one to generate $g_{i}^{(2)}(\tau)$. In figure 5 , the comparison is made between $\left\langle g_{i}^{(2)}(\tau)\right\rangle$ estimated by the direct method and that by using the Siegert relation:

$$
g_{i}^{(2)}(\tau) \approx 1+\left|g^{(1)}(\tau)\right|^{2}
$$

As expected in this particular setting (Jakeman 1974), the two methods lead to the same result (i.e. the Siegert relation holds) for large $P_{\text {move }}$ only. Even in this case, the behaviour depicted in figure 5 cannot be reproduced by the classical MC algorithms.

\section{Discussion and conclusions}

In the present work, it has been shown that the $\mathrm{MC}$ data can serve to extract more information than usually done. To that aim, MC data are kept in their natural representation and used to directly compute $i(t)$. Once $i(t)$ is obtained, any algorithm used in a real LDF instrument can be tested and validated. This eliminates the tedious procedure of generating a specific 
(often approximate) model, accepting the MC data (e.g. for $P_{i}(\omega)$ or $g_{i}^{(2)}(\tau)$ ), each time a new algorithm is considered. A possible elegant and computationally efficient manner to store the large amount of MC data is to use a sparse representation. In fact, a very large number of photon packets do not reach the detector and these values can be represented by zeros, allowing a sparse representation.

The present approach also eliminates the problem of $\hat{h}_{W}\left(\delta \omega_{n}\right)$ construction. In fact, as was explained in section 2.4.2, $\hat{h}_{W}\left(\delta \omega_{n}\right)$ is the estimator of an 'ideal' $f(\Delta \omega)$ distribution describing the MC data. Thus, for given MC data, there is in principle only one best $\hat{h}_{W}\left(\delta \omega_{n}\right)$, i.e. the bin width $\delta \omega$ can be found by applying an adequate algorithm that optimizes a given criterion (see e.g. Davies et al (2009)). This means that $\delta \omega$ has an optimal value which depends on the MC data only. This is in contradiction with the example from section 3.3 where we needed to predefine different $\delta \omega$ values ('classical' procedure) to simulate the effect of different choices of $T_{i}$. Moreover, the MC data have a very large discontinuity at $\omega=0$ that makes the histogram construction problematic in the neighbourhood of the $\mathrm{dc}$ bin. The direct approach proposed here avoids these potential problems.

The different examples also demonstrate another effect that can be described only with the direct method, i.e. the dependence of $g_{i}^{(2)}(\tau \approx 0)$ on $P_{\text {move }}$ (see e.g. figure 5). The very short interoptode spacing was chosen on purpose to obtain a very low number of interactions of photons with moving red blood cells. In fact, with the present interoptode spacing, the volume visited by the photons can be estimated to be less than $1 \mathrm{~mm}^{3}$. This implies that for a low concentration of red blood cells (low $P_{\text {move }}$ ), the number of scattering events (with a moving cell) is also low (i.e. only low scattering orders). As pointed out also by Jakeman (1974), the Siegert relation does not necessarily hold in this case and this implies $g_{i}^{(2)}(\tau \approx 0)$ smaller than 2 . An example has been shown in figure 5 , where only large values of $P_{\text {move }}$ (i.e. presence of high scattering orders) will lead to $g_{i}^{(2)}(\tau \approx 0) \approx 2$ and to the validity of the Siegert relation.

In practical terms, the geometry of the model utilized in the present tutorial represents a simplified LDF instrument for skin tissue monitoring (for complex skin models, see e.g. Meglinski and Matcher (2002), Meglinski et al (2008)). However, for the medical and biomedical community the interest for LDF goes far beyond this (important) application, and larger interoptode spacing, allowing one to reach brain, muscle, etc, is often considered. In fact, all tissues need nutrients, oxygen, mechanisms allowing one to eliminate metabolic wastes, etc. In this context, blood circulation plays a fundamental role and its dysfunction may lead to severe pathologies and eventually to tissue necrosis. This is why LDF and in general near-infrared-based techniques are extremely interesting for physicians even in the case of tissues such as the skeleton (Binzoni et al 2006a). It is essential to note that from the previous examples, it is not clear if the Siegert relation still holds for large interoptode spacing (e.g. 3$4 \mathrm{~cm}$ ) and for a biological tissue having a low red blood cell concentration (e.g. bone, tendon, white adipose tissue), as is usually assumed. This may be an intriguing question for future studies because to our knowledge, all the classical analytical models for $g_{i}^{(2)}(\tau)$, utilized in practice, implicitly make the assumption of the validity of the Siegert relation. The proposed direct method shows that $g_{i}^{(2)}(\tau \approx 0)<2$ does not necessarily imply bad $(\beta<1)$ spatial coherence (actually $\beta$ simplifies in the expression for $g_{i}^{(2)}(\tau)$; equation (8) and that another phenomenon related to the random nature of $\Phi(\omega)$ must be taken into account.

Actually, together with spatial coherence, $\Phi(\omega)$ and coherence length of the laser, other parameters may influence $g_{i}^{(2)}(\tau)$ and its amplitude $g_{i}^{(2)}(\tau \approx 0)$. In a real experiment, the light may be detected through an optical fibre and the specific choice of this fibre (e.g. single-mode, multi-mode) influence $g_{i}^{(2)}(\tau)$ (Rička 1993). Detector nonlinearities (due to dead-time, afterpulsing) also have a non-negligible effect on the acquired optical signals and 
on $g_{i}^{(2)}(\tau)$ (Flammer and Rička 1997). Moreover, the biological tissue itself can manifest another property that has not been taken into account in the present MC examples, i.e. the modulation of light polarization (Kuzmin and Meglinski 2007, Meglinski et al 2005), and that influences $g_{i}^{(2)}(\tau)$ as well. Taking into account these parameters in the generation of $i(t)$ will strongly improve the future model.

There is no doubt that there is a feverish activity in the LDF domain and that new approaches to the hardware (e.g. integrated systems, path-length-resolved LDF, full field laser Doppler imaging) regularly appear in the literature (for an up-to-date review, see Rajan et al (2009)). This implies the development of new theoretical models allowing one to adapt functional algorithms for this instrumentation. Among the last theoretical discoveries one may also cite the laser-Doppler spectrum decomposition, allowing one to estimate the speed distribution of the red blood cells (Humeau et al 2007, Wojtkiewicz et al 2009), and the demonstration of the non-negligible influence of the speckle phenomenon on the laser-Doppler imaging signal (Rajan et al 2008). These are important topics in LDF that need to be further understood and developed. Better knowledge of $i(t)$, a signal common to all the LDF methods, will certainly allow us to bring new insight.

In conclusion, we hope that the series of tutorial examples have demonstrated the interesting potentialities of the proposed direct method and that this will help to develop and test new approaches of LDF.

\section{Acknowledgments}

The authors would like to thank the 'Faculté de Médecine' of Geneva for the Mimosa grant that allowed the computer cluster to be set up. This work was also supported in part (last author, DVDV) by the Center for Biomedical Imaging (CIBM).

\section{References}

Ahn H, Johansson K, Lundgren O and Nilsson G E 1987 In vivo evaluation of signal processors for laser Doppler tissue flowmeters Med. Biol. Eng. Comput. 25 207-11

Binzoni T, Leung T S, Giust R, Rüfenacht D and Gandjbakhche A H 2008a Light transport in tissue by 3D Monte Carlo: influence of boundary voxelization Comput. Methods Prog. Biomed. 89 14-23

Binzoni T, Vogel A, Gandjbakhche A H and Marchesini R 2008b Detection limits of multi-spectral optical imaging under the skin surface Phys. Med. Biol. 53 617-36

Binzoni T, Leung T S, Courvoisier C, Giust R, Tribillon G, Gharbi T and Delpy D T 2006a Blood volume and haemoglobin oxygen content changes in human bone marrow during orthostatic stress J. Physiol. Anthropol. 25 1-6

Binzoni T, Leung T S, Rüfenacht D and Delpy D T 2006b Absorption and scattering coefficient dependence of laser-Doppler flowmetry models for large tissue volumes. Phys. Med. Biol. 51 311-33

Binzoni T and Van De Ville D 2008 Full-field laser-Doppler imaging and its physiological significance for tissue blood perfusion Phys. Med. Biol. 53 6673-94

Boas D A 1996 Diffuse photon probes of structural and dynamical properties of turbid media: theory and biomedical applications Dissertation University of Pennsylvania

Bonner R and Nossal R 1981 Model for laser Doppler measurements of blood-flow in tissue Appl. Opt. 20 2097-107

Bowman and Azzalini 1997 Applied Smoothing Techniques for Data Analysis: The Kernel Approach with S-Plus Illustrations (New York: Oxford University Press)

Briers J D 2001 Laser Doppler, speckle and related techniques for blood perfusion mapping and imaging Physiol. Meas. 22 R35-66

Briers J D 2007 Laser speckle contrast imaging for measuring blood flow Opt. Appl. 37 139-52

Cohen-Tannoudji C, Dupon-Roc J and Grynberg G 2004 Photons and Atoms. Introduction to Quantum Electrodynamics (Weinheim: Wiley)

Cummins H Z and Swinney H L 1970 Light beating spectroscopy Progress in Optics vol 8 ed E Wolf (Amsterdam: North-Holland) pp 133-200 
Davies P L, Gather U, Nordman D J and Weinert H 2009 A comparison of automatic histogram constructions ESAIM: PS 13 181-96

de Mul F F M, Koelink M H, Kok M L, Harmsma P J, Greve J, Graaff R and Aarnoudse J G 1995 Laser Doppler velocimetry and Monte Carlo simulations on models for blood perfusion in tissue Appl. Opt. 34 6596-611

Draijer M, Hondebrink E, van Leeuwen T and Steenbergen W 2009 Twente Optical Perfusion Camera: system overview and performance for video rate laser Doppler perfusion imaging Opt. Express 17 3211-25

Fairs S L 1988 Observations of a laser Doppler flowmeter output made using a calibration standard Med. Biol. Eng. Comput. 26 404-6

Flammer I and Rička J 1997 Dynamic light scattering with single-mode receivers: partial heterodyning regime Appl. Opt. 36 7508-16

Forrester A T 1961 Photoelectric mixing as a spectroscopic tool J. Opt. Soc. Am. 51 253-9

Glauber R J 1965 Optical coherence and photon statistics Quantum Optics and Photon Statistics (Les Houches Lecture Notes 1964) ed C de Witt, A Blandin and C Cohen-Tannudji (New York: Gordon and Breach) pp 63-185

Humeau A, Steenbergen W, Nilsson H and Strömberg T 2007 Laser Doppler perfusion monitoring and imaging: novel approaches Med. Biol. Eng. Comput. 45 421-35

Jaillon F, Skipetrov S E, Li J, Dietsche G, Maret G and Gisler T 2006 Diffusing-wave spectroscopy from head-like tissue phantoms: influence of a non-scattering layer Optics Express 14 10181-94

Jakeman E 1974 Photon correlation Correlation and Light Beating ed H Z Cummins and E R Pike (New York: Plenum) pp 75-149

Kienle A 2001 Non-invasive determination of muscle blood flow in the extremities from laser Doppler spectra Phys. Med. Biol. 46 1231-44

Koelink M H, de Mul F F M, Greve J, Graaff R, Dassel A C M and Aarnoudse J G 1994 Laser Doppler blood flowmetry using two wavelengths: Monte Carlo simulations and measurements Appl. Opt. 33 3549-58

Kuzmin V L and Meglinski I V 2007 Coherent effects of multiple scattering for scalar and electromagnetic fields: Monte-Carlo simulation and Milne-like solutions Opt. Commun. 273 307-10

Lamb W and Scully M 1969 The photoelectric effect without photons Polarisation, Matiere et Rayonnement ed Société Fraçaise de Physique (Paris: Presses Universitaires de France) pp 363-9

Leahy M J, de Mul F F, Nilsson G E and Maniewski R 1999 Principles and practice of the laser-Doppler perfusion technique Technol. Health Care 7 143-62

Liebert A, Leahy M and Maniewski R 1995 A calibration standard for laser-Doppler perfusion measurements Rev. Sci. Instrum. 66 5169-73

Liebert A, Lukasiewicz P, Boggett D and Maniewski R 1999 Optoelectronic standardization of laser Doppler perfusion monitors Rev. Sci. Instrum. 70 1352-4

Mandel L, Sudarshan E C G and Wolf E 1964 Theory of photoelectric detection of light fluctuations Proc. Phys. Soc. 84 435-44

Meglinski I, Kirillin M, Kuzmin V and Myllylä R 2008 Simulation of polarization-sensitive optical coherence tomography images by a Monte Carlo method. Opt. Lett. 33 1581-3

Meglinski I V, Kuzmin V L, Churmakov D Y and Greenhalgh D A 2005 Monte Carlo simulation of coherent effects in multiple scattering Proc. R. Soc. A $46143-53$

Meglinski I V and Matcher S J 2002 Quantitative assessment of skin layers absorption and skin reflectance spectra simulation in the visible and near-infrared spectral regions Physiol. Meas. 23 741-53

Nilsson G E, Tenland T and Oberg P A 1980 Evaluation of a laser Doppler flowmeter for measurement of tissue blood flow IEEE Trans. Biomed. Eng. 27 597-604

Nussenzveig H M 1973 Introduction to Quantum Optics (New York: Gordon and Breach)

Obeid A N 1993 In vitro comparison of different signal processing algorithms used in laser Doppler flowmetry Med. Biol. Eng. Comput. 31 43-52

Pines D J, Weitz J X, Zhu J X and Herbolzheimer E 1990 Diffusive-wave spectroscopy: dynamic light scattering in the multiple scattering limit J. Phys. France 51 2101-27

Raabe A et al 2009 Laser Doppler imaging for intraoperative human brain mapping NeuroImage 44 1284-89

Rajan V, Varghese B, Van Leeuwen T G and Steenbergen W 2008 Influence of tissue optical properties on laser Doppler perfusion imaging, accounting for photon penetration depth and the laser speckle phenomenon J. Biomed. Opt. 13024001

Rajan V, Varghese B, van Leeuwen T G and Steenbergen W 2009 Review of methodological developments in laser Doppler flowmetry Lasers Med. Sci. 24 269-83

Rička J 1993 Dynamic light scattering with single-mode and multimode receivers Appl. Opt. 32 2860-75

Serov A and Lasser T 2005 High-speed laser Doppler perfusion imaging using an integrating CMOS image sensor Opt. Express 13 6416-28 
Serov A, Steenbergen W and de Mul F 2002 Laser Doppler perfusion imaging with a complimentary metal oxide semiconductor image sensor Opt. Lett. 27 300-2

Serov A, Steinacher B and Lasser T 2005 Full-field laser Doppler perfusion imaging and monitoring with an intelligent CMOS camera Opt. Express 13 3681-9

Skipetrov S E and Meglinski I V 1998 Diffusing-wave spectroscopy in randomly inhomogeneous media with spatially localized scatterer flows J. Exp. Theor. Phys. 86 661-5

Smits G J, Roman R J and Lombard J H 1986 Evaluation of laser-Doppler flowmetry as a measure of tissue blood flow J. Appl. Physiol. 61 666-72

Soelkner G, Mitic G and Lohwasser R 1997 Monte Carlo simulations and laser Doppler flow measurements with high penetration depth in biological tissuelike head phantoms Appl. Opt. 36 5647-54

Steenbergen W and de Mul F 1998a Application of a novel laser Doppler tester including a sustainable tissue phantom Proc. SPIE 3252 14-25

Steenbergen W and de Mul F F M 1998b New optical tissue phantom and its use for studying laser Doppler blood flowmetry Proc. SPIE 3196 12-23

Stern M D 1985 Laser Doppler velocimetry in blood and multiply scattering fluids: theory Appl. Opt. 24 1968-86

Vennemann P, Lindken R and Westerweel J 2007 In vivo whole-field blood velocity measurement techniques Exp. Fluids 42 495-511

Wang L, Jacques S L and Zheng L 1995 MCML-Monte Carlo modeling of light transport in multi-layered tissues Comput. Methods Prog. Biomed. 47 131-46

Wilson B C and Adam G A 1983 Monte-Carlo model for the absorption and flux distributions of light in tissue Med. Phys. 10 824-30

Wojtkiewicz S, Liebert A, Rix H, Zołek N and Maniewski R 2009 Laser-Doppler spectrum decomposition applied for the estimation of speed distribution of particles moving in a multiple scattering medium Phys. Med. Biol. 54 679-97 